\title{
Technè
}

La science au service de l'histoire de l'art et de la préservation des biens culturels

48 | 2019

Les couleurs de l'Antique

\section{Un nouvel emploi du cobalt identifié sur un vase en albâtre de la XVIII dynastie inscrit au nom d'Amenhotep III découvert à Ougarit (Syrie)}

A new use of cobalt identified on an alabaster vase inscribed with the name of Amenhotep III discovered in Ugarit (Syria)

\section{Valérie Matoïan et Anne Bouquillon}

\section{OpenEdition}

Journals

Édition électronique

URL : https://journals.openedition.org/techne/2846

DOI : $10.4000 /$ techne. 2846

ISSN : 2534-5168

Éditeur

C2RMF

Édition imprimée

Date de publication : 31 décembre 2019

Pagination : 150-157

ISBN : 978-2-11-152831-4

ISSN : $1254-7867$

\section{Référence électronique}

Valérie Matoïan et Anne Bouquillon, « Un nouvel emploi du cobalt identifié sur un vase en albâtre de la XVIII dynastie inscrit au nom d'Amenhotep III découvert à Ougarit (Syrie) », Technè [En ligne], 48 2019, mis en ligne le 01 juillet 2021, consulté le 10 avril 2022. URL : http://journals.openedition.org/ techne/2846 ; DOI : https://doi.org/10.4000/techne.2846

\section{(ब) $\Theta \Theta$}

La revue Technè. La science au service de l'histoire de l'art et de la préservation des biens culturels est mise à disposition selon les termes de la Licence Creative Commons Attribution - Pas d'Utilisation Commerciale - Pas de Modification 4.0 International. 


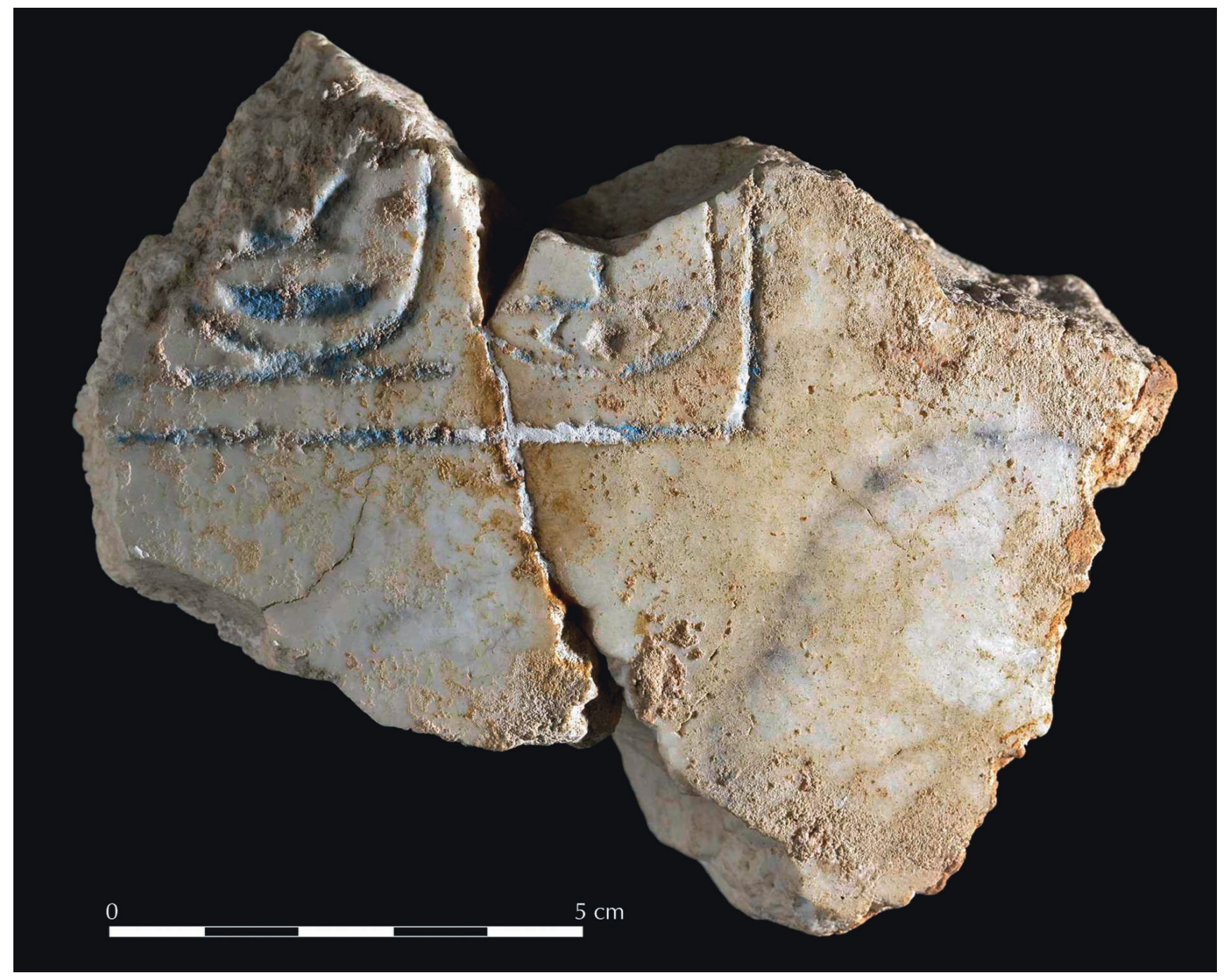

Fig. 1. Fragment de vase en albâtre calcaire de Ras Shamra, Louvre, inv. AO 27127 (H. 10,4 cm, L. 11,5 cm). (c) Cliché C2RMF/A. Maigret. Infographie mission de Ras Shamra/G. Devilder. 
Valérie Matoïan

Anne Bouquillon

\section{Un nouvel emploi du cobalt identifié sur un vase en albâtre de la XVIII ${ }^{\mathrm{e}}$ dynastie inscrit au nom d'Amenhotep III découvert à Ougarit (Syrie)}

A new use of cobalt identified on an alabaster vase inscribed with the name of Amenhotep III discovered in Ugarit (Syria)

\section{En hommage à Sandrine Pagès-Camagna}

Sandrine Pagès-Camagna fut l'un des acteurs de la recherche ici présentée, en sa qualité de spécialiste du bleu égyptien. Nous lui dédions cet article en témoignage de notre amitié et de notre estime.

Résumé. Des incrustations bleues soulignant les hiéroglyphes de deux fragments d'un vase en albâtre calcaire inscrit au nom du pharaon Amenhotep III (Louvre, inv. AO 27127) découvert à Ougarit ont été étudiées par des méthodes non-destructives. L'analyse révèle que du cobalt a été utilisé comme élément colorant, probablement sous la forme spinelle proche de celle identifiée pour les pigments des céramiques égyptiennes («blue-painted pottery») de la même époque.

Mots-clés. Ougarit, Bronze récent, matières vitreuses, vaisselle en albâtre, Égypte, cobalt, PIXE (Particle-induced X-ray emission), LIR (Luminescence dans le proche infrarouge).
Abstract. Blue inlays highlighting the hieroglyphs on two fragments of a calcite alabaster vase inscribed with the name of Pharaoh Amenhotep III (Louvre, inv. AO 27127) and discovered in Ugarit were studied using non-destructive methods. The analysis revealed that cobalt was used as the colorant, probably in the spinel form close to that identified as the pigments in bluepainted pottery from the same period.

Keywords. Ugarit, Late Bronze Age, vitreous materials, alabaster vessel, Egypt, cobalt, PIXE (Particle-induced X-ray emission), VIL (Visible-induced luminescence).
Parmi les royaumes du Levant septentrional de l'âge du Bronze, celui d'Ougarit (Syrie) est l'un des mieux documentés pour la période allant du $\mathrm{XIV}^{\mathrm{e}}$ au début du XII ${ }^{\mathrm{e}}$ siècle avant J.-C. Son histoire à l'âge du Bronze récent est liée à celle des grands royaumes de l'époque : le Mitanni, l'Égypte du Nouvel Empire et le Hatti dont Ougarit est un vassal à partir de la seconde moitié du XIV siècle. Les données archéologiques et textuelles livrent l'image d'un royaume florissant, occupant une place privilégiée sur la côte méditerranéenne, au carrefour des voies de circulation internationales entre la Méditerranée orientale et le Proche-Orient. Sa capitale, qui porte le même nom, Ougarit, est devenue un site de référence pour l'étude des civilisations urbaines et palatiales du ProcheOrient et de la Méditerranée orientale.

Les recherches sur le site de Ras Shamra, très vite identifié comme la capitale d'Ougarit, et sur celui de Minet el-Beida où était implanté son port principal (antique Mahadu), ont commencé en 1929. L'exposition «Ougarit, une cité méditerranéenne ", présentée au musée du Louvre du 6 juillet au 2 décembre 2019, marque donc le quatre-vingt dixième anniversaire du début des fouilles ${ }^{1}$.

\section{Contexte archéologique de l'étude}

L'exploration a mis au jour un abondant matériel archéologique, illustrant de nombreux artisanats. À partir des années 1980, des programmes d'analyses archéométriques portant sur différentes catégories d'objets découverts à Ras Shamra et Minet el-Beida ont été réalisés au Laboratoire de recherche des musées de France (devenu depuis lors Centre de recherche et de restauration des musées de France). Les arts du feu ont fait l'objet d'un intérêt tout particulier², avec notamment le développement de plusieurs recherches sur les matières vitreuses.

Valérie Matoïan, chargée de recherche au CNRS, UMR 7192 Proclac (CNRS - Collège de France) (valerie.matoian@college-de-france.fr). Anne Bouquillon, ingénieur de recherche, C2RMF - Institut de Recherche Chimie Paris, PSL Research University, Chimie ParisTech-CNRS, UMR 8247 (anne.bouquillon@culture.gouv.fr). 


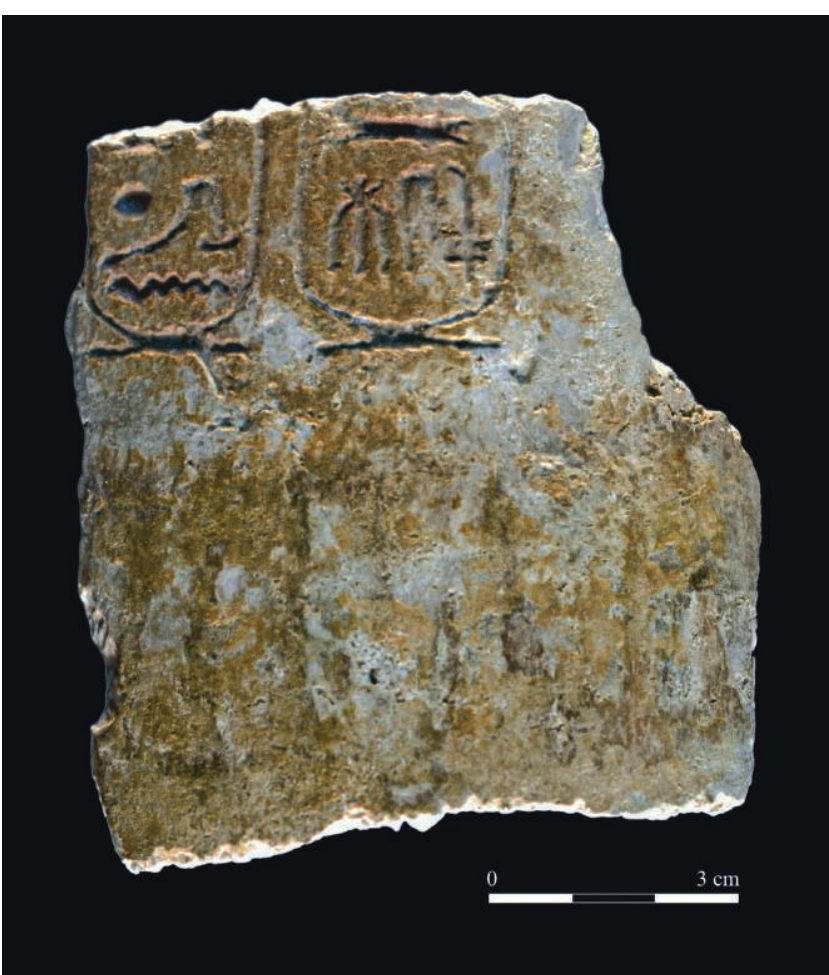

Fig. 2. Fragment de vase en albâtre calcaire au nom d'Amenhotep IV (RS 15.202, musée national de Damas 4155), Ras Shamra.

(c) Mission de Ras Shamra, cliché V. Matoïan, infographie G. Devilder.

Ougarit a en effet livré l'un des corpus d'objets en matières vitreuses parmi les plus importants du ProcheOrient pour la période du Bronze récent. Plus de 17000 faïences, un millier d'objets en verre, environ 250 en bleu égyptien et moins d'une trentaine en céramique argileuse à glaçure ont été répertoriés ${ }^{3}$. Le répertoire typologique est d’une grande variété : vases, figurines, éléments de parure, pommeaux, pions de jeu, cachets, sceaux-cylindres, scarabées, éléments d'incrustation...

Le matériel d'Ougarit a tout d'abord été étudié dans le cadre d'un vaste programme sur les faïences de l'Orient ancien, initié par A. Caubet et A. Kaczmarczyk à partir des collections du département des Antiquités orientales du musée du Louvre ${ }^{4}$. À cette recherche initiale ont succédé quatre opérations portant respectivement sur la céramique argileuse à glaçure ${ }^{5}$, le bleu égyptien ${ }^{6}$, la faïence monochrome bleu-gris ${ }^{7}$ et le verre ${ }^{8}$. Ces recherches, initiées par V. Matoïan, ont été menées en collaboration avec A. Bouquillon, I. Biron, S. Pagès-Camagna et avec la participation d'Y. Coquinot et d'A. Leclaire. Elles se sont inscrites dans le cadre d'une collaboration entre le C2RMF, le musée du Louvre et la Mission archéologique syro-française de Ras Shamra-Ougarit ${ }^{9}$.

Les objectifs premiers étaient de mieux caractériser les matériaux et d'essayer de définir l'origine des productions (importation ou production locale). En montrant la présence à Ougarit d'une série bien documentée d'objets en faïence monochrome bleu-gris colorés au cobalt, en permettant

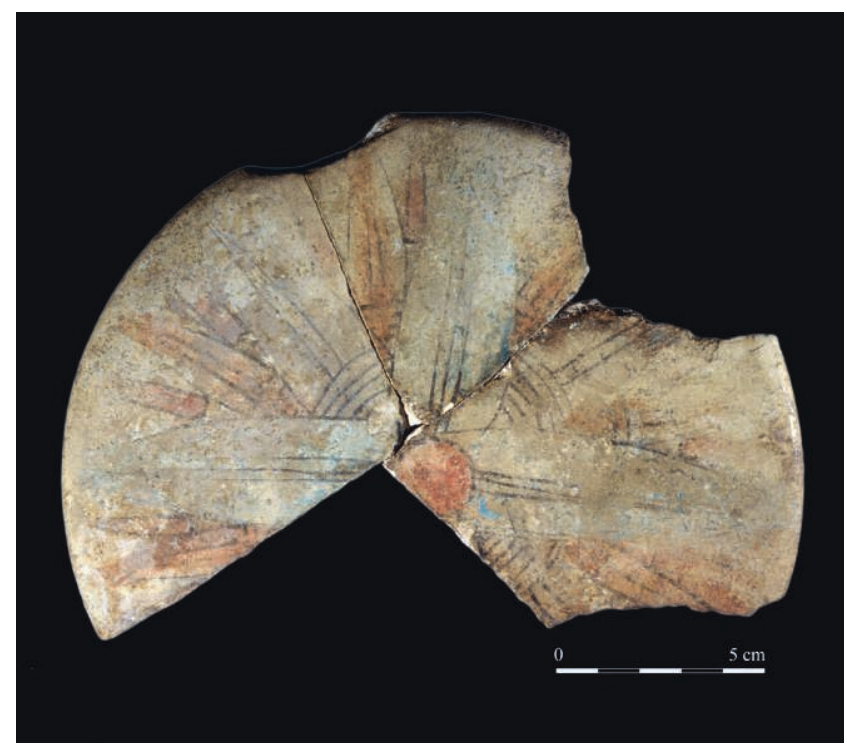

Fig. 3. Couvercle en calcaire à décor de corolle florale polychrome (RS 17.157, musée national de Damas 4495), Ras Shamra. (C) Mission de Ras Shamra, cliché V. Matoïan, infographie G. Devilder.

d'identifier un nouvel emploi du bleu égyptien comme colorant d'un corps de faïence, en révélant l'une des premières utilisations de verre incolore, ces travaux ont apporté des résultats novateurs.

Nous avons repris à partir de 2014 les dossiers du bleu égyptien et de la faïence monochrome bleu gris en menant des analyses complémentaires sur plusieurs séries d’objets conservés au département des Antiquités orientales du musée du Louvre, principalement des perles, des sceaux-cylindres et des pains de bleu égyptien brut. La sélection comportait aussi deux fragments jointifs d'un vase en pierre de Ras Shamra dont la panse présente des incrustations de matière bleue. L'œuvre est répertoriée sous le numéro d'inventaire de musée AO 27127 (fig. 1). Ni le numéro d'inventaire de fouille, ni la provenance précise de l'objet ne sont connus ${ }^{10}$. Les fragments appartiennent à un vase jubilaire au nom du pharaon Amenhotep III (env. 1392-1354 av. J.-C.) de la $\mathrm{XVIII}^{\mathrm{e}}$ dynastie égyptienne ${ }^{11}$. Il fait partie d'une série restreinte de vases égyptiens en albâtre calcaire ${ }^{12}$ inscrits aux noms de plusieurs pharaons du Nouvel Empire égyptien (Amenhotep III, Amenhotep IV-Akhenaton, Horemheb, Ramsès II) ; retrouvés à Ougarit dans le Palais royal et dans de grandes demeures de la cité, ces vases sont interprétés comme des cadeaux de Pharaon en témoignage des bonnes relations entre l'Égypte et le royaume d'Ougarit ${ }^{13}$.

Deux autres vases en albâtre au nom d'Amenhotep III ont été identifiés à Ougarit : RS 17.58 (Damas DO 4465) et RS 11.329 (Louvre 84 AO 548). Le premier a été mis au jour 


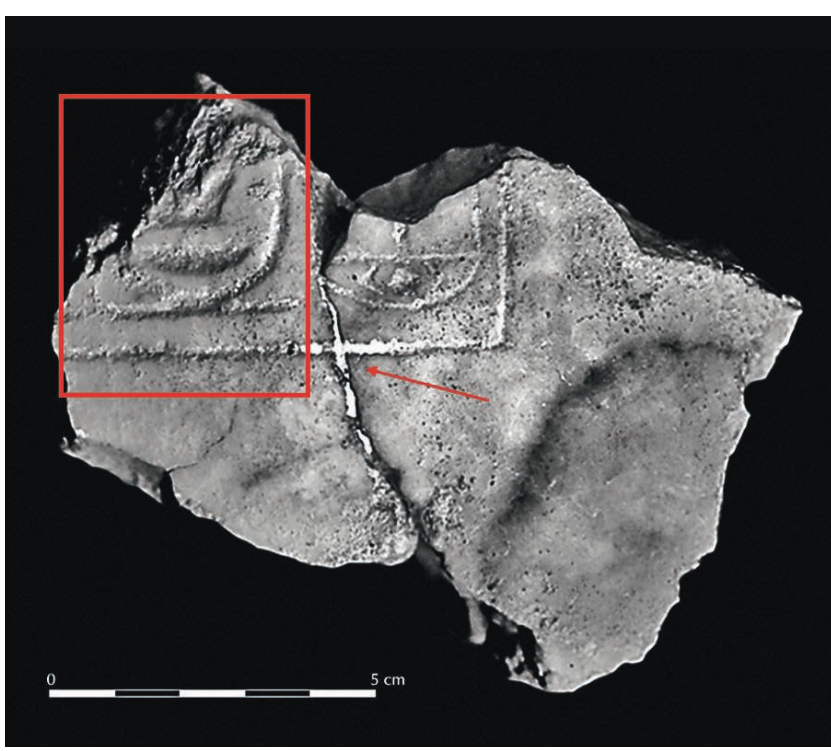

Fig. 4. Vase Louvre AO 27127 : observation de la fluorescence dans le domaine de l'infrarouge $(910 \mathrm{~nm})$. La zone étudiée dans le fragment à gauche sur la photo ne présente aucune fluorescence (cadre rouge). Les fluorescences au niveau de la cassure ou de la limite du cartouche à droite (voir flèche) ne sont pas expliquées pour le moment. (C) Cliché C2RMF/A. Maigret. Infographie mission de Ras Shamra/ G. Devilder.

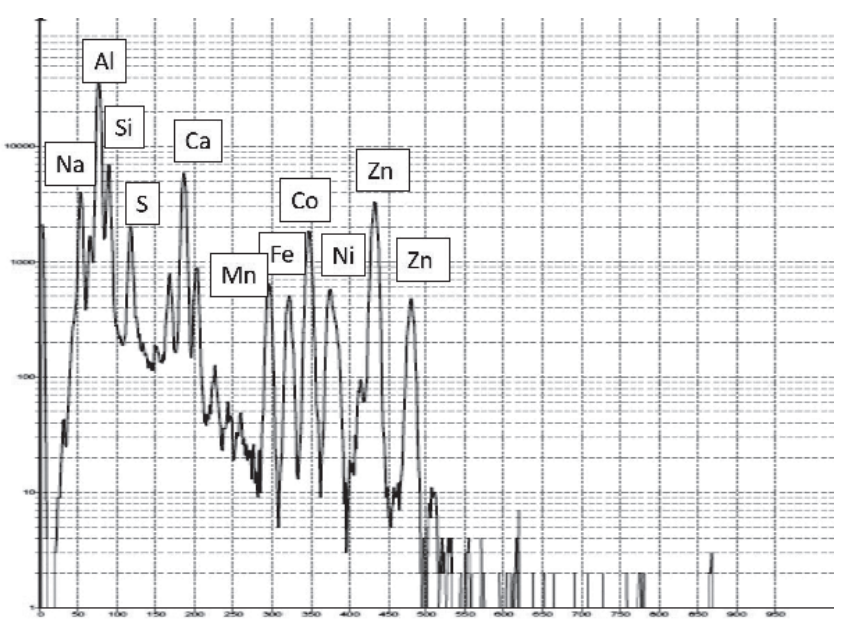

lors de la fouille du Palais royal, tandis que le second provient du secteur dit «Ville Basse ouest ${ }^{14}$ ». Sept scarabées portant le nom de ce pharaon ont également été retrouvés à Ras Shamra, parmi lesquels un grand scarabée commémoratif aux noms du pharaon et de son épouse, la reine Tiy ${ }^{15}$.

Les vestiges d'incrustations ou de peintures de couleur bleue sont rares à Ougarit. Jusqu'à présent, les pièces les mieux documentées sont des éléments en verre bleu (en forme de boucle, de baguette ou de pétale) qui faisaient probablement partie, sous forme d'incrustations, du décor de meubles en bois ${ }^{16}$.

Des traces de matière bleue (observées au moment des fouilles mais aujourd'hui disparues) ont été signalées par les fouilleurs sur certains objets en ivoire considérés comme des productions locales ${ }^{17}$.

Enfin, plusieurs catégories de vases présentent les restes d'un décor bleu (incrusté ou peint). Il s'agit :

- de vases en albâtre calcaire égyptiens. Les deux fragments Louvre AO 27127 appartiennent à cette dernière catégorie. On signalera aussi quatre autres vases incomplets, deux aux noms d'Amenhotep IV (RS 15.202, fig. 2 et RS 15.203) et deux aux noms de Ramsès II (RS 15.201 et RS 34.30)18. RS 34.30 présente à la fois un décor gravé, avec des restes de matière bleue, et un décor peint, en noir, rouge et bleu.

- de céramiques importées d'Égypte portant encore les traces d'un décor peint en bleu ${ }^{19}$.

- de couvercles en calcaire à décor de corolle florale polychrome. Un spécimen mis au jour dans le Palais royal conserve encore une partie de son décor peint avec des zones de couleur bleue (RS 17.157, Damas 4495) ${ }^{20}$ (fig. 3).

\section{L'analyse du pigment bleu}

Plusieurs auteurs ont proposé, sur la base d'observations visuelles, que le pigment utilisé pour les matières bleues observables sur le matériel retrouvé à Ougarit soit du bleu égyptien $^{21}$. Or, en ce qui concerne la matière bleue incrustée sur le vase Louvre AO 2712722, l'imagerie scientifique ainsi que les analyses en laboratoire montrent que tel n'est pas le cas.

En effet, G. Verri (2009) a montré que le bleu égyptien - plus particulièrement les cristaux de cuprorivaïte $\left(\mathrm{CaCuSi}_{4} \mathrm{O}_{10}\right)$ qu'il contient - émet une fluorescence caractéristique dans le domaine de l'infrarouge $(910 \mathrm{~nm})$ quand il est éclairé par une source lumineuse rouge $(610 \mathrm{~nm})$. Pour la matière bleue du vase Louvre AO 27127, aucune fluorescence n'est observée dans la partie gauche, là où la coloration est la plus franche (fig. 4), il s'agit donc d'un autre matériau colorant. En revanche, une fluorescence apparaît sur le cliché au niveau de la cassure médiane et à droite près d'un hiéroglyphe. Elle correspond à un matériau blanc (fig. 1) non encore identifié à ce niveau de l'étude (collage ?...).

Dans un second temps, nous avons analysé l'incrustation avec l'accélérateur de particules AGLAE, par PIXE, de façon totalement non-destructive. Nous avons pu éviter que le calcium constitutif de l'albâtre vienne "polluer » la composition 


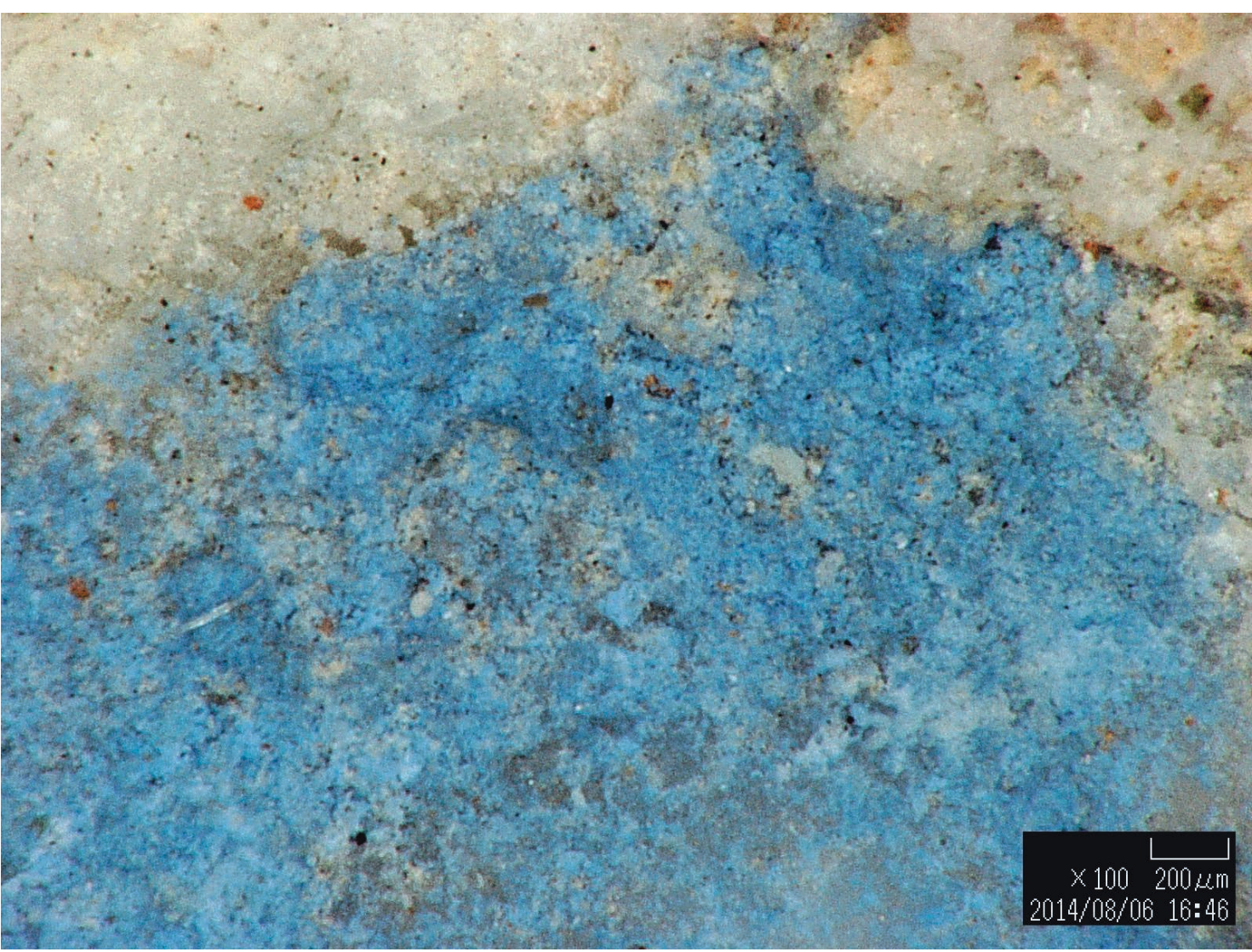

Fig. 6. Détail de l'incrustation bleue du vase AO 27127. (c C2RMF/S. Pagès-Camagna.

chimique de la matière bleue en focalisant très précisément le faisceau de protons dont le diamètre est proche de $50 \mu \mathrm{m}$ sur l'incrustation soulignée dans la figure 4. Les résultats montrent la présence du cobalt, associé à de l'aluminium, élément largement majoritaire, du zinc, du fer, du manganèse et du nickel (fig. 5). Les concentrations relatives de chaque élément sont détaillées dans le tableau 1.

L'utilisation de cobalt est fréquente dans les matériaux vitreux du $\mathrm{II}^{\mathrm{e}}$ millénaire en Égypte et à Ougarit. Cet oxyde métallique aux pouvoirs colorants puissants se retrouve dans les verres et les glaçures des faïences ou sur des céramiques. À Ougarit, le cobalt est notamment l'élément colorant des glaçures de tous les objets en faïence monochrome bleu-gris que nous avons analysés ${ }^{23}$. Il a aussi été détecté dans un petit bloc de verre bleu foncé retrouvé dans le Palais royal et dont la composition est proche de celle des verres égyptiens du Nouvel Empire ${ }^{24}$. Dans ces matériaux pourtant, les teneurs en cobalt n'excèdent pas $1 \% \mathrm{CoO}$; on y retrouve cependant les mêmes éléments chimiques associés au cobalt, en quantités certes très inférieures à celles détectées dans les incrustations du vase Louvre AO 27127, mais dont les rapports restent assez proches (tableau 2). Les associations des différents éléments chimiques signent les conditions géochimiques de dépôts des gisements. Ici, elles semblent correspondre à celles des aluns cobaltifères des oasis du désert occidental égyptien ${ }^{25}$. Cette hypothèse d'une origine égyptienne de l'élément colorant est confortée par la typologie des objets concernés : la plupart des faïences bleu-gris, tout comme le fragment de vase ici étudié, présentent de fortes affinités typologiques et stylistiques avec les productions mises au jour en Égypte.

L'observation d'un détail de l'incrustation bleue du vase (fig. 6) et la composition chimique montrent nettement que le pigment coloré n'est pas du verre bleu pilé que l'on aurait mélangé à une charge. C'est un pigment spécifique. Dans l'Égypte du Nouvel Empire, en parallèle à son utilisation comme colorant dans divers matériaux vitreux (faïence, fritte, verre), le cobalt a aussi servi pour les décors peints appliqués sur certaines céramiques destinées aux élites ${ }^{26}$.

Tableau 1. Composition chimique de l’incrustation bleue du vase AO 27127 (données PIXE exprimées en \% massiques d'oxydes)

\begin{tabular}{|l|l|l|l|l|l|l|l|l|l|l|l|l|l|l|l|l|}
\cline { 2 - 11 } \multicolumn{1}{c|}{} & $\mathrm{Na}_{2} \mathrm{O}$ & $\mathrm{MgO}^{\prime}$ & $\mathrm{Al}_{2} \mathrm{O}_{3}$ & $\mathrm{SiO}_{2}$ & $\mathrm{SO}_{3}$ & $\mathrm{Cl}$ & $\mathrm{K}_{2} \mathrm{O}$ & $\mathrm{CaO}$ & $\mathrm{TiO}_{2}$ & $\mathrm{MnO}$ & $\mathrm{Fe}_{2} \mathrm{O}_{3}$ & $\mathrm{CoO}$ & $\mathrm{NiO}$ & $\mathrm{ZnO}$ & $\mathrm{SrO}$ & $\mathrm{Cu0}$ \\
\hline $\begin{array}{l}\text { Louvre } \\
\text { A0 27127 }\end{array}$ & 8,11 & 2,26 & 51,94 & 13,87 & 3,40 & 0,03 & 0,45 & 4,70 & 0,04 & 0,82 & 0,75 & 2,86 & 1,07 & 9,67 & 0,01 & 0,03 \\
\hline
\end{tabular}


Tableau 2. Comparaison des rapports $\mathrm{MgO} / \mathrm{CoO}, \mathrm{MnO} / \mathrm{CoO}$, $\mathrm{Fe}_{2} \mathbf{0}_{3} / \mathrm{CoO}, \mathrm{NiO} / \mathrm{CoO}, \mathrm{ZnO} / \mathrm{CoO}, \mathrm{Al}_{2} \mathrm{O}_{3} / \mathrm{CoO}$

Données issues de l'article de Shortland et al., 2006. Les lignes en italiques correspondent aux valeurs minimales et maximales mentionnées par les auteurs.

\begin{tabular}{|l|l|l|l|l|l|l|}
\cline { 2 - 7 } \multicolumn{1}{c|}{} & $\mathrm{MgO} / \mathrm{C} 00$ & $\mathrm{MnO} / \mathrm{C}_{0} 0$ & $\mathrm{Fe}_{2} \mathrm{O}_{3} / \mathrm{C}_{0} 0$ & $\mathrm{ZnO} / \mathrm{C}_{0} 0$ & $\mathrm{NiO} / \mathrm{C}_{0}$ & $\mathrm{Al}_{2} \mathrm{O}_{3} / \mathrm{C}_{0} 0$ \\
\hline Vase & 0,8 & 0,3 & 0,3 & 3,4 & 0,4 & 18,1 \\
\hline Makalta $*$ & 1,0 & 0,5 & 0,6 & 3,2 & 0,6 & 14,7 \\
\hline & $0,67-1,94$ & $0,12-0,33$ & $0,32-0,71$ & $2,47-5,11$ & $0,31-0,74$ & $11,1-20,89$ \\
\hline Amarna * & 2,4 & 0,4 & 1,3 & 3,3 & 0,8 & 17,5 \\
\hline & $1,50-3,20$ & $0,23-0,43$ & $0,97-2,45$ & $1,91-4,37$ & $0,7-1,1$ & $15,3-20,6$ \\
\hline
\end{tabular}

Connue sous l'appellation de «blue-painted pottery », cette céramique est un marqueur de la culture matérielle égyptienne du Nouvel Empire. Plusieurs phases de production ont été reconnues, du milieu de la XVIII ${ }^{\mathrm{e}}$ dynastie (règne d'Amenhotep II) au milieu de la $\mathrm{XX}^{\mathrm{e}}$ dynastie (règne de Ramsès IV). Des ateliers ont existé dans la région memphite et dans la région thébaine. Cette céramique décorée est particulièrement bien attestée sous le règne d'Amenhotep III, avec un centre de production à Malkata ${ }^{27}$. Les peintures bleues sur céramique ont été largement étudiées depuis les années $1980^{28}$ et on sait maintenant que le pigment est un aluminate de cobalt ${ }^{29}$, un spinelle de la forme $\mathrm{CoAl}_{2} \mathrm{O}_{4}$ contenant aussi d'autres éléments métalliques $(\mathrm{Zn}, \mathrm{Fe} . .$.$) , obtenu à partir$ d'un traitement thermique et éventuellement chimique des aluns. La comparaison des données chimiques de l'incrustation avec celles publiées par A. Shortland, C. A. Hope et M. S. Tite en 2006 montre qu'il s'agit très probablement du même type de pigment (tableau 1).

Sur les céramiques, ce type de décor bleu est, selon les différents chercheurs cités précédemment, systématiquement posé avant cuisson, de même que les décors noirs (spinelle $\mathrm{Fe} / \mathrm{Mn}$ ) et rouges (hématite). De l'alun riche en cobalt serait posé directement sur un engobe argileux enrichi en gypse ; le tout est porté à une température comprise entre $800^{\circ} \mathrm{C}$ et $1000^{\circ} \mathrm{C}$, nécessaire au développement de la couleur et à la cristallisation des phases spinelles. Dans le cas des décors colorés posés après cuisson, des analyses ont montré que la couleur bleue pouvait être due à l'emploi de bleu égyptien ${ }^{30}$, tout comme pour les enduits peints architecturaux ${ }^{31}$.

La matière constitutive du vase est ici de l'albâtre calcaire et on ne peut imaginer une cuisson de l'objet qui aurait détérioré l'aspect de l'œuvre. Il faut donc envisager une autre préparation spécifique du pigment qui puisse être appliqué à froid sur un objet.

W. Noll (1981), cité par Y. Abe (2009), propose un protocole en trois étapes : 1 . dissolution de l'alun dans l'eau et obtention de solutions de sulfates ; 2. précipitations d'hydroxydes métalliques par ajout d'alcalins à cette solution (natron ?) ; 3. cuisson du précipité obtenu entre 900 et $1000^{\circ} \mathrm{C}$. Le produit obtenu sera alors posé sur l'objet.
Dans les analyses de l'incrustation du vase AO 27127 (tableau 1), les teneurs importantes en sodium $\left(8 \% \mathrm{Na}_{2} \mathrm{O}\right)$ pourraient alors s'expliquer par l'utilisation de natron $\left(\mathrm{Na}_{2} \mathrm{CO}_{3} \bullet 10 \mathrm{H}_{2} \mathrm{O}\right)$. Nous n'avons pas trouvé mention en bibliographie de creusets ayant contenu ce type de préparation ou de pains de matières brutes qui pourraient corroborer cette recette.

Notre hypothèse à ce stade de la recherche est donc qu'un pigment à base de cobalt, sous forme de spinelle, a été mis en œuvre. Il est prévu de réaliser maintenant d'autres études, par diffractométrie des rayons X tout d'abord, puis par spectrométrie Raman, pour valider cette piste et trouver comment ce pigment a été posé.

\section{Conclusion}

La culture matérielle de la civilisation ougaritique comprend un grand nombre, d'une part, d'œuvres égyptiennes importées et, d'autre part, de productions artistiques locales (ou régionales) témoignant de l'influence égyptienne. Ougarit a livré un des corpus les plus importants d'egyptiaca du Levant parmi lesquels des pièces aux noms de pharaons du Moyen et du Nouvel Empire (majoritairement des statues et de la vaisselle en albâtre). Pour la première fois, l'analyse d'un vase en albâtre a révélé l'emploi d'un pigment à base de cobalt pour la matière bleue incrustée dans le décor en creux de la panse. Cette découverte permet d'envisager de nouvelles pistes de recherche sur les couleurs en Égypte et au Proche-Orient ancien, ainsi que dans le domaine de la science des matériaux.

Un premier programme d'envergure portant spécifiquement sur les incrustations de couleur bleue devra être envisagé. Par ailleurs, des expérimentations comparables à celles menées par C. Chauffeton ${ }^{32}$ et G. Wallez pour l'étude d'un autre spinelle alumineux contenant du cobalt découvert au XIX ${ }^{\mathrm{e}}$ siècle de notre ère et largement utilisé à la manufacture de Sèvres seraient importantes pour mieux préciser les conditions thermiques et les protocoles de fabrication de ce matériau. 


\section{Notes}

1. Salle des actualités du département des Antiquités orientales (commissariat: V. Matoïan et M. Pic) : Matoïan, Pic, 2019

2. Des études ont également porté sur les roches (voir Yon, 1991) et les métaux.

3. Matoïan, 2000.

4. Bouquillon, Caubet, Kaczmarczyk, Matoïan, 2007. Quelques objets en bleu égyptien avaient été analysés antérieurement par C. Lahanier.

5. Matoïan, Bouquillon, 1999.

6. Matoïan, Bouquillon, 2000.

7. Bouquillon, Matoïan, 2007.

8. Biron, Matoïan, Henderson, Evans, 2012.

9. Les études en laboratoire ont porté sur des pièces conservées dans les musées en Syrie, exportées temporairement grâce à l'autorisation de la Direction générale des antiquités et des musées de Syrie et, en France, au musée du Louvre. Nous remercions vivement le département des Antiquités orientales pour son soutien constant, en particulier mesdames A. Caubet, B. André-Salvini, M. Pic et S. Cluzan.

10. Caubet, 1991, p. 230 : RS 1-11. [116].

11. Lagarce, 2008, p. 272-273, fig. 11.

12. Icart, Chanut, Matoïan, 2008, p. 184-185.

13. Lagarce, 2008 ; Lagarce, Othman, Grimal, 2013.

14. Matoïan, 2020 (à paraître).

15. Lagarce-Othman, 2017.

16. Matoïan, 2014.

17. Gachet-Bizollon, 2007, p. 22.

18. Lagarce, du Puytisson-Lagarce, 2008, Lagarce, 2008 ; p. 266 et 271 ; Bounni et al., 1998, p. 71.

19. Lagarce, 2008, p. 160.

20. Icart, Chanut, Matoïan, 2008, p. 167-168, fig. 4, pl. IX, 1 et 2.

21. Bounni et al., 1998, p. 77 ; Matoïan, Bouquillon, 2000, p. 987-988 ; Lagarce, 2008, p. 263.

22. Documentation C2RMF, $\mathrm{n}^{\circ}$ de dossier C2RMF 73634.

23. Bouquillon, Matoïan, 2007.

24. Matoïan, Bouquillon, 2007.

25. Voir Kaczmarczyk, Hedges, 1983 ; Kaczmarczyk, 1986.

26. Le cobalt n'est pas attesté dans les peintures murales égyptiennes pour lesquelles le pigment est le bleu égyptien (Lee, Quirke, 2000, p. 108 et 111).

27. Sur cette catégorie céramique, voir notamment : Bourriau et al., 2005 ; Aston, 2011.

28. Noll, 1981; Shortland, 2000; Shortland $e t$ al., 2006 ; Abe, 2009

29. Shortland, 2000 , p. 48.

30. Lee, Quirke, 2000, p. 108 et 111.

31. Abe, 2009

32. Chauffeton, 2018.

\section{Bibliographie}

Abe Y., Nakai I., Takahashi K., Kawai N., Yoshimura S., 2009, "On-site analysis of archaological artefacts excavated from the site on the outcrop at Northwest Saqqara, Egypt, by using a newly developed portable fluorescence spectrometer and diffractometer", Analytical and Bioanalytical Chemistry 395 , p. $1987-1996$

Aston D. A., 2011, "Blue-painted Pottery of The Late Eighteenth Dynasty, The Material from the Tomb of Maya and Merit at Saqqara", Cahiers de la Céramique égyptienne 9, p. 1-35.

Biron I., Matoïan V., Henderson J., Evans J., 2012, "Scientific analysis of glass beads from Ras Shamra-Ugarit (Syria)”, dans D. Ignatiadou et A. Antonaras (eds.), Annales du $18^{e}$ congrès de l'Association Internationale pour l'Histoire du Verre, Thessalonique (20-25 septembre 2009), Thessalonique, p. 27-32.

Bounni A., Lagarce E. et J., 1998, Ras Ibn Hani, I. Le palais nord du Bronze récent. Fouilles 1979-95, synthèse préliminaire, Bibliothèque Archéologique et Historique CLI, Beyrouth.

Bouquillon A., Caubet A., Kaczmarczyk A. Matoïan V., 2007, Faïences et matières vitreuses de l'Orient ancien, éditions Snoeck - Musée du Louvre éditions, Grand-Paris.

Bouquillon A., Matoïan V., 1998, « Deux perles syriennes en "bleu égyptien” savoir-faire local ou matériau importé ?", Techne n 7 , p. 21-22.

Bouquillon A., Matoïan V., 2007, « Les faiences à glaçure monochrome bleugris d'Ougarit (Syrie) ", dans J.-P. Goyon et C. Cardin (éds.), Actes du Neuvième Congrès International des Égyptologues, Grenoble, 2004, Leuven/ Paris/Dudley, MA, p. 207-220.

Bourriau J., Aston D., Raven M. J., van Walsem R., 2005, The Memphite Tomb of Horemheb Commander-in-chief of Tut'ankhamun, III, The New Kingdom Pottery, Egypt Exploration Society, Londres.

Caubet A., 1991, « Répertoire de la vaisselle de pierre. Ougarit 1929-1988 ", dans M. Yon (éd.), Arts et industries de la pierre, Ras Shamra-Ougarit VI, ERCADPF, Paris, p. 205-264.

Gachet-Bizollon J., 2007, Les ivoires d'Ougarit, Ras Shamra-Ougarit XVI, Éditions Recherche sur les Civilisations, Paris.

Grimal N., 2013, « Diplomatie et écriture : à propos des inscriptions égyptiennes d'Ougarit ", dans P. Bordreuil, F. ErnstPradal, M. G. Masetti-Rouault, H. Rouillard-Bonraisin (éds.), Les écritures mises au jour sur le site antique d'Ougarit (Syrie) et leur déchiffrement 1930-2010, Commémoration du quatre-vingtième anniversaire du déchiffrement de l'alphabet cunéiforme de Ras Shamra-Ougarit, AIBL, Paris, p. 187-202.

Icart J.-C., Chanut C., Matoïan V., 2008, « Le matériel en pierre du Palais royal d'Ougarit: diagnose, nomenclature, provenance et usage ", dans V. Matoïan (dir.), Le mobilier du Palais royal d'Ougarit, Ras Shamra-Ougarit XVII, Maison de l'Orient et de la Méditerranée, Lyon, p. 157-190.

Kaczmarczyk A., 1986, "The Source of Cobalt in Ancient Egyptian Pigments", dans J. S. Olin, M. J. Blackman (eds.),
Proceedings of the 24th International Archaeometry Symposium, Smithsonian Institution Press, Washington, p. 369376.

Kaczmarczyk A., Hedges R.E.M., 1983, An Analytical Survey of Egyptian Faience from Predynastic to Roman Times, Aris and Philips Ltd, Warminster.

Lagarce B., 2008, "Réexamen des monuments du Palais royal d'Ougarit inscrits en hiéroglyphes égyptiens conservés au musée national de Damas », dans V. Matoïan (dir.), Le mobilier du Palais royal d'Ougarit, Ras Shamra-Ougarit XVII, Maison de l'Orient et de la Méditerranée, Lyon, p. 261-280.

Lagarce-Othman B., 2013, «Un nouveau vase inédit d'Horemheb ", dans V. Matoïan et M. Al-Maqdissi (éds.), Études ougaritiques III, Ras ShamraOugarit XXI, Paris/Leuven/Walpole, MA, p. 347-364.

Lagarce-Othman B., 2017, « Les scarabées d'Amenhotep III et de Tiy à Ougarit ", dans V. Matoïan (dir.), Archéologie, patrimoine et archives. Les fouilles anciennes à Ras Shamra et à Minet el-Beida, I, Ras Shamra-Ougarit XXV, Éditions Peeters, Leuven, p. 165-186.

Lagarce J., du Puytisson-Lagarce E., 2008, "Remarques sur le matériel égyptien et égyptisant de Ras Shamra ("maison aux Albâtres ») et de Ras Ibn Hani à la lumière de données récentes sur la chronologie de la fin d'Ugarit ", dans M. Bietak et E. Czerny (eds.), The Bronze Age in the Lebanon, Studies on Archaeology and Chronology of Lebanon, Syria and Egypt, Vienne, p. 153-164.

Lee L., Quirke S., 2000, "Painting materials", dans P. T. Nicholson et I. Shaw (eds.), Ancient Egyptian materials and Technology, Cambridge, p. 104-120.

Matoïan V., 2020 (à paraître), “Ægyptiaca from Ugarit and their contexts: inscribed royal objects dated to the Middle and New Kingdom", Semitica.

Matoïan V., Bouquillon A., 1999, « La céramique argileuse à glaçure de Ras Shamra-Ougarit », Syria 76, p. 57-82.

Matoïan V., Bouquillon A., 2000, «Le "bleu égyptien” à Ras Shamra-Ougarit (Syrie) ", dans P. Matthiae, A. Enea, L. Peyronel et F. Pinnock (eds.), Proceedings of the First International Congress on the Archaeology of Ancient Near East, Rome 1998, Rome, p. 983-1004.

Matoïan V., Bouquillon A., 2007, «Matériaux vitreux découverts à Tell Ashara-Terqa (chantiers E et F, quinzième campagne) - II - Un lingot de matière vitreuse (TQ 15.411) de l'époque du royaume de Hana ", dans J.-C. Margueron, O. Rouault et P. Lombard (éds.), Akh Purattim 2, série "Akh Purattim - Les rives de l'Euphrate ", Maison de l'Orient et de la Méditerranée - Ministère des Affaires étrangères, Lyon, p. 199-207.

Matoïan V., Pic M., 2019, « À la découverte du royaume d'Ougarit ", Grande Galerie, Le Journal du Louvre 48, p. 78-79.

Noll W., 1981, "Mineralogy and technology 
of the painted ceramics of ancient Egypt", dans M. J. Hugues (ed.), Scientific Studies in Ancient Ceramics, British Museum Occasional Papers 19, p. $143-154$.

Pagès-Camagna S., 1998, « Pigments bleu et vert égyptiens en question : vocabulaire et analyses ", dans S. Colinart et M. Menu (éds.), La couleur dans la peinture et l'émaillage de l'Égypte ancienne, Actes de la table ronde, Ravello, mars 1997 , p. 163-175.

Shortland A. J., 2000, Vitreous Materials at Amarna, The production of glass and faience in the 18th Dynasty Egypt, BAR International Series 827, Oxford.
Shortland A. J., Hope C. A., Tite M. S., 2006 , "Cobalt blue-painted pottery from 18th Dynasty Egypt", Geological Society, London, Special Publications 257, p. 91-99.

Verri G., 2009, "The spatially resolved characterization of Egyptian blue, Han blue and Han purple by photo-induced luminescence digital imaging", Analytical and Bioanalytical Chemistry 394, no. 4 (June 2009):1011-1021. doi: 10.1007/s00216-009-2693-0.

Yon M. (éd.), 1991, Arts et industries de la pierre, Ras Shamra-Ougarit VI, ERCADPF, Paris.

\section{Documents inédits}

Chauffeton C., 2018, Étude et caractérisation des pigments historiques de la Manufacture de Sèvres - Autour du Bleu Thénard, mémoire de Master II, Institut de Recherche de Chimie Paris.

Matoïan V., 2000, Ras Shamra-Ougarit et la production des matières vitreuses au ProcheOrient au II ${ }^{e}$ millénaire av. J.-C., thèse de doctorat, université de Paris I Panthéon Sorbonne.

Matoïan V., 2014, Ugarit, l'Égypte et les Phéniciens. Manuscrit inédit, dossier d'HDR, université Paris I-Panthéon Sorbonne. 